\title{
Molecular Landscape of Non-Muscle Invasive Bladder Cancer
}

\author{
Edward M. Messing* \\ University of Rochester Medical Center, Rochester, NY, USA
}

The molecular alterations of muscle-invasive (MI) urothelial cancer (UC) have been studies extensively by the Cancer Genome Atlas (TCGA) [1]. A consensus classification not dissimilar to that used for breast cancer, with luminal and basal subtypes, among others, reflecting various degrees of tumor aggressiveness and responsiveness to systemic chemotherapy, has been developed. This classification has great translational potential, which will likely be applied for clinical purposes soon. While this work addresses the most lethal form of bladder cancer, it is still only applicable for roughly $25 \%$ of patients newly diagnosed with this disease. What about the $75 \%$ of patients with non-muscle invading (NMI) UC, particularly the considerable majority with low grade (LG) tumors? Most of these patients are never going to die from bladder cancer, but will experience frequent recurrences and undergo lifelong cystoscopic monitoring, contributing greatly to costs of care and patient morbidity and inconvenience. Several recent articles have tried to develop a molecular classification of NMI tumors and while not all methods and patient subgroups (by standard histology and staging) were the same, some common themes have arisen.

Hurst and co-workers [2] building on earlier work, in which the majority of specimens came from patients with high grade, stage, T1 or T2 UC, [3] reported on the mutational landscape of primarily low grade NMI bladder cancers. These investigators

\footnotetext{
${ }^{*}$ Correspondence to: Edward M. Messing, University of Rochester Medical Center, Rochester, NY, USA. Tel.: +1 585275 3345; E-mail: Edward.messing@urmc.rochester.edu.
}

studied 140 patients with grade 1 and 2 , stage Ta UCs, although with re-examination, $39 \%$ were considered high grade (HG) by the 2004 WHO criteria [4]. They reported that virtually all tumors would be considered to have a luminal signature based on the TCGA classification for MIBC. Two subgroups, one with few chromosomal abnormalities and a high frequency of mutations in chromatin remodeling genes (termed GS1), and the other (GS2), with loss of chromosome $9 \mathrm{q}$, a higher mutation rate, and marked upregulation of mammalian target of rapamycin complex 1 (mTORC1) and its subsequent downstream signaling (primarily because TSC1, mTORC1's transcriptional regulator, was lost with chromosome $9 \mathrm{q}$ deletion). GS2 tumors had a higher mutational number and because of increased mTORC1 activity, greater lipid and nucleotide synthesis and increased aerobic glycolysis. Patients with GS2 tumors had generally more aggressive malignancies, having more high grade tumors and a (non-significantly) worse recurrence free survival [2]. Both groups had many tumors with activating mutations of fibroblast growth factor receptor 3 (FGFR3) and primarily inactivating mutations of KDM6A (a chromatin remodeling demethylase) [2, 5]. Although only a preliminary finding, KDM6A mutations were found more frequently in female patients, the only genetic change that appeared to be associated with gender.

While Hurst and colleague [2] used chromosomal and mutational analyses, as well as protein and gene expression analyses, the GS1 group was very similar to the urobasal A class described by Sjodahl, et al. [6], and the UROMOL class 1 by Hedegaard et al. [7], using primarily gene expression data. In terms of 
gene expression, the GS2 group was very similar to the UROMOL class 2 [7].

Because of inclusion of more high grade cases and much larger case numbers $(\mathrm{N}=476$ rather than 140) than Hurst, et al. [2] studied, Hedegaard and colleagues [7] demonstrated that UROMOL class 2 cancers had a much higher chance of subsequently progressing to muscle invading disease than UROMOL class 1 NMI UCs.

While these distinctions (GS1 vs GS2, Urobasal A/UROMOL class 1 vs UROMOL class 2) just like for MIBC have not been used clinically yet, it is likely that GS2/UROMOL 2 tumors should receive more aggressive therapy, and possibly mTORC1 inhibitors (e.g. evirolimus) or agents that block its downstream signaling, including those that inhibit cholesterol/lipid synthesis and transport or glycolysis. Perhaps even intravesical administration of these agents can be tested.

However, it should be noted that there are limitations in both interpreting the results of these studies as well as in implementing them. For all of these studies on NMI UC (and for the TCGA), frozen tissue specimens were utilized, something that is not part of standard clinical practice, and it's unclear whether classifications, particularly for gene expression signatures can be duplicated with very small formalin fixed specimens. Secondly, if multiple tumors were present, it's not clear whether only one was selected for these analyses or whether specimens were pooled, etc. Thirdly, when looking at recurrence and progression, the impact of treatment (e.g. immediate post transurethral resection [TURBT] instillations of chemotherapy, repeat TURBTs, subsequent courses of intravesical chemo - or immuno-therapy) also was not analyzed.

Despite these limitations, these studies represent a huge advance in our understanding of (particularly low grade) NMI UC and should be investigated at least as companion projects, in ongoing clinical trials in this group of patients.

\section{REFERENCES}

[1] Cancer Genome Atlas Research Network. Comprehensive molecular characterization of urothelial bladder carcinoma. Nature 2014;507:315-24.

[2] Hurst CD, Alder O, Platt FM, et al. Genomic subtypes of noninvasive bladder cancer with distinct metabolic profile and female gender bias in KDM6A mutation frequency. Cancer Cell 2017;32:701-15.

[3] Hurst CD, Platt FM, Taylor CF, et al. Novel tumor subgroups of urothelial carcinoma of the bladder defined by integrated genomic analysis. Clin Can Res 2012;18:5865-77.

[4] Eble JN, et al. World Health Organization Classification of Tumors. Pathology and genetics of tumors of the urinary system and male genital organs. IARC Press, Lyon, PR, 2004.

[5] Ler LD, Ghosh S, Chai X, et al. Loss of tumor surpressor KDM6A amplifies PRC2-regulated transcriptional repression in bladder cancer and can be targeted through inhibition of EZH2. Science Transl Med 2017;9:eaa 8312.

[6] Sjohdahl G, Lauss M, Lovgren K, et al. A molecular taxonomy for urothelial cancer. Clinc Cancer Res 2012;18:3377-86.

[7] Hedegaard J, Lamy P, Nordentoft I, et al. Comprehensive transcriptional analysis of-early stage urothelial carcinoma. Cancer Cell 2016;30:27-42. 